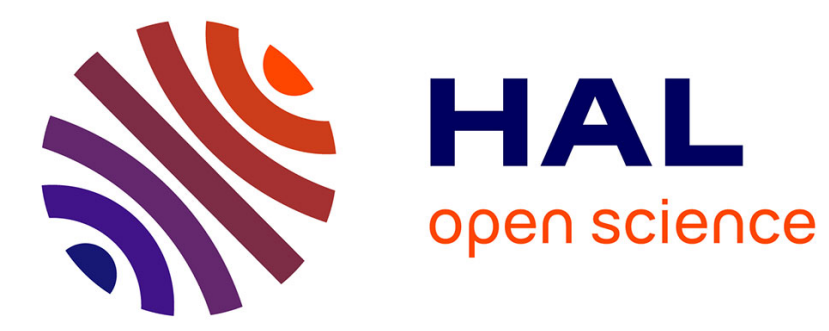

\title{
Sound propagation in colloidal systems
}

\author{
L. Ye, J. Liu, P. Sheng, J. Huang, D. Weitz
}

\section{To cite this version:}

L. Ye, J. Liu, P. Sheng, J. Huang, D. Weitz. Sound propagation in colloidal systems. Journal de Physique IV Proceedings, 1993, 03 (C1), pp.C1-183-C1-196. 10.1051/jp4:1993116 . jpa-00251559

\section{HAL Id: jpa-00251559 https://hal.science/jpa-00251559}

Submitted on 1 Jan 1993

HAL is a multi-disciplinary open access archive for the deposit and dissemination of scientific research documents, whether they are published or not. The documents may come from teaching and research institutions in France or abroad, or from public or private research centers.
L'archive ouverte pluridisciplinaire HAL, est destinée au dépôt et à la diffusion de documents scientifiques de niveau recherche, publiés ou non, émanant des établissements d'enseignement et de recherche français ou étrangers, des laboratoires publics ou privés. 


\title{
Sound propagation in colloidal systems
}

\author{
L. YE, J. LIU, P. SHENG, J.S. HUANG and D.A. WEITZ
}

Exxon Research and Engineering Co., Rt. 22 E, Annandale, NJ 08801, U.S.A.

\begin{abstract}
We study the propagation of sound in complex colloidal systems. By combining Brillouin scattering with ultrasonic techniques, we measure the dispersion in the acoustic propagation over three decades in frequency. Acoustic propagation is sensitive to the bulk compressibility of the medium, and probes new structural and dynamic properties of the colloidal system. We study two colloidal systems. The first is a system of inverted micelles or microemulsions, where the droplet size is significantly smaller than the wavelength of the sound. By measuring the dispersion of the sound velocity as a function of droplet volume fraction, we identify an increased rigidity of the system at high frequencies. The increase in the modulus scales as $\left(\phi-\phi_{c}\right)^{\tau}$, where $\phi$ is the volume fraction of droplets and $\phi_{c}$ is a critical volume fraction. This is consistent with rigidity percolation. The second system we study consists of a suspension of hard sphere colloids whose diameter is comparable to the wavelength of sound. We measure the dispersion curve for the phonons in this system at different volume fractions of spheres. A new acoustic excitation is found when the wavelength of the sound is comparable to the sphere diameter. This acoustic excitation possesses unusual properties and is attributed to a surface excitation that can propagate coherently between adjacent spheres.
\end{abstract}

Nous etudions la propagation du son dans les systems colloidaux complexes. En combinant la diffusion Brillouin avec les techniques ultrasoniques, nous mesuront la dispersion de la propagation acoustique sur trois decades en frequence. La propagation acoustique est sensible a la compressiblite du millieu, et explore des proprietes nouvelles des systemes colloidaux. Nous etudions deux systemes colloidaux. Le premier est un systeme de micelles inverses ou la taille des micelles est plus petite que la longueur d'onde du son. En mesurant la dispersion de la celerite du son en fonction de la fraction volumique en micelles, nous identifiont une rigidite croissante du systeme a haute frequence. Le module croit comme $\left(\phi-\phi_{c}\right)^{\tau}$, ou $\phi$ est la fraction volumique en micelles et $\phi_{c}$ est un fraction volumique critique, et $\tau$ un exposant en accord avec une rigidite decrite par la theorie de la percolation. Le deuxieme systeme est compose de spheres dures dont le diametre est voisin de la longueur d'onde du son. Nous mesuront la courbes de dispersion des phonons a differentes fractions volumiques. Une nouvelle excitation acoustique est trouvee quand le longueur d'onde du son est comparable a la taille des spheres. Ce mode possede des propriete unusuelles et est attrubue a un excitation de surface qui peut se propager entre les spheres. 


\section{INTRODUCTION}

The propagation of sound through colloidal systems can probe new properties that characterize both the structure and the dynamics of the suspension. The presence of the colloidal particles in the suspension results in structures whose length scales are larger than molecular, resulting in a modification of the elastic properties of colloidal system. A study of the propagation as the wavelength of the sound is varied can provide a measure of the elastic properties of these structures, and thus a measure of the characteristics of the structures. Moreover, a study of the propagation as the frequency of the sound is varied can provide important new information about the dynamics of these structures. Measurement of the sound propagation provides information that is distinct from, but complementary to, that provided by the more commonly performed scattering experiments. Traditional scattering experiments probe the structure of the colloidal system. By contrast, the propagation of sound is sensitive to the connectivity of the structure in the suspension. Thus the propagation of sound provides a measure of one of the most critical properties of the suspension, and provides a complement to scattering studies of the structure of colloidal suspensions.

In this paper, we summarize the results of studies of sound propagation in two different colloidal systems. In one case, the wavelength of the sound is much greater than the size of the colloidal particles in the suspension, and the measurements therefore probe larger length scale structures that results from the dynamics of the particles. In the second case, the wavelength of the sound is comparable to the size of the colloidal particles, and the measurements therefore probe the consequences of the presence of the particles on the propagation of sound through the suspension. In both cases, we use Brillouin scattering to measure the propagation of sound in the colloidal suspension. In this technique, the light scattered at some scattering wave vector, $q$, is spectrally resolved with an interferometer, thereby probing the light scattered from the thermally excited sound waves with the same wave vector, $q$. A measure of the frequency of these sound waves yields their velocity. In one case, we also complement the Brillouin measurements with ultrasonic measurements of the sound propagation, providing a measure of the behavior at much lower frequencies and longer wavelengths.

The first system we study $[1,2]$ is comprised of micelles or microemulsions formed from AOT (sodium di-2-ethylhexylsulfosuccinate), oil and water [3 - 6]. In the absence of water, the AOT molecules form uniformly sized inverted micelles. Moreover, as the volume fraction of the AOT is varied, the size of the micelles remains constant, and only their volume fraction changes. Upon addition of water, the micelles swell into microemulsions, with the water inside the droplets and the oil the continuous phase. However, again the size of the droplets remains constant as their volume fraction is varied. In both cases, the size of the colloidal particles is much smaller than the wavelength of sound. We measure the dispersion in the sound velocity, $v$, over three and a half decades by combining ultrasonic and Brillouin techniques. The dispersion is also measured as the volume fraction, $\phi$, of the micelle or microemulsion droplets is varied. In addition, we determine the effects of the length of the hydrocarbon chain of the oil molecules and the size of the microemulsion droplets on the propagation of the sound. We find that the velocity of sound in the system increases as the volume fraction of droplets increases, indicating that the surfactant shells of the droplets are more rigid than the continuous oil medium, and thus have a higher sound velocity. In addition, as the frequency of the sound is increased, the dispersion with $\phi$ is even greater.

We interpret this effect as resulting from longer range networks formed by the droplets because of a weak attractive interaction between them. However, because the attractive force between the droplets is so weak, these longer range networks are transient, and the frequency of the sound must be above the inverse of the lifetime of these networks in order to be sensitive to their existence. When this is true, these networks contribute an additional rigidity because they can support shear, provided their size is sufficiently large that they span the system. The difference in the sound velocities at low and high frequencies provides a measure of the additional shear modulus due to the networks. We find that this additional rigidity exhibits a power-law scaling with volume fraction as $\phi$ approaches a critical value, $\phi_{c}$, characteristic of a percolation behavior. We call this behavior dynamic rigidity percolation. The use of different oils with varying lengths of hydrocarbon chains modifies the strength of the interaction, thereby changing the characteristic time scale of the existence of the transient networks. In addition, the larger size of the microemulsion droplets increases the strength of the attractive interaction because the contact area between the droplets grows. However, the intrinsic rigidity of the droplets decreases upon addition of increasing amounts of water. These physical concepts provide a consistent account of all our observations.

The second system we study $[7,8]$ is comprised of monodisperse PMMA (polymethylmethacrylate) colloidal particles suspended in a mixture of dodecane and carbon disulfide that provides a nearly exact match to the index of refraction of the colloidal particles, eliminating all multiple scattering. The colloidal particles are sterically stabilized by a thin coating of adsorbed polymer, about $15 \mathrm{~nm}$ thick, 
resulting in a hard sphere interaction between the particles. The diameter of the particles used is comparable to the wavelength of the light, and by varying the scattering angle, we are able to probe the propagation of sound through this colloidal system as the wavelength is changed from much larger than the particle diameter, to comparable to the particle diameter to much smaller than the particle diameter. While the index of refraction for light of the particles and the solvent are closely matched, there remains a large impedance mismatch between the particles and the solvent for sound waves, resulting in very strong scattering of the sound. We find that when the wavelength of the sound is much larger than the particle diameter, there is a single propagating sound wave, whose velocity increases as the volume fraction of particles, $\phi$, is increased. However, as the wavelength of the sound becomes comparable to the particle diameter, we observe a new propagating mode, whose velocity is slower than that of the sound modes in either of the two pure materials, the fluid or the solid. Moreover, the velocity of this new mode decreases as the volume fraction of the particles is increased. We interpret this mode as a surface wave that can exist only when the sound wavelength is comparable to, or smaller than, the sphere diameter. When the wavelength becomes smaller than the diameter, this mode reverts to a Stoneley wave, an interfacial sound wave that exists at the interface between a solid which possesses a shear modulus and a fluid. The mode decays in the fluid between the spheres, but, at sufficiently high $\phi$, can nevertheless propagate between adjacent spheres. We present a measurement of the full dispersion curves for sound propagation in a suspension of hard sphere colloids for different volume fractions.

\section{MICELLES AND MICROEMULSIONS}

We begin by considering the behavior of the inverted micelles, which consist of 22 AOT molecules with the close packed hydrophilic head groups forming a solid core, while the hydrocarbon tail groups extend in to the solvent oil $[9,10]$. The diameter of the micelles is $a \approx 30 \AA$, and remains fixed as the volume fraction of the micelles is varied. There is a weak short range attractive interaction between the micelles $[11,12]$. This is probably entropic in nature, resulting from the entanglement of the hydrocarbon tail groups of two micelles. Since the tail groups are already correctly aligned by their configuration in the micelles, there is no decrease in entropy when they become entangled. By contrast, the solvent molecules can adopt any orientation required to fit in between the tail groups of the surfactant molecules in a micelle. This results in a weak, entropically driven, attractive interaction, whose energy depends on temperature and on the size of the solvent molecules, since larger molecules have a larger loss in entropy in fitting between the surfactant tail groups.

We measure both the speed and the damping of the longitudinal sound as the volume fraction of the micelles is varied. To determine the dispersion over a very large frequency range, we combine both ultrasonic and Brillouin measurements. For the lower frequencies, we use time-of-flight ultrasonic

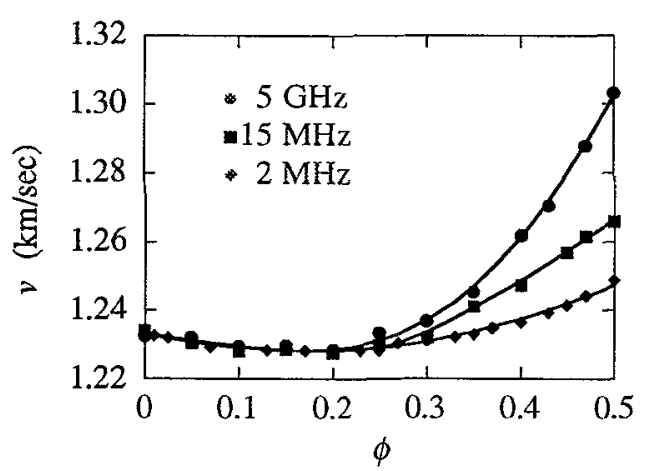

Fig. 1: Sound velocity as a function of volume fraction for micelles in decane measured with several different frequencies: $2 \mathrm{MHz}, 15 \mathrm{MHz}$ and $5 \mathrm{GHz}$. The solid lines through the data are fits to the effective medium model, using isolated spheres for the surfactant phase for the lowest frequency, and a random, connected network that supports shear for the higher frequencies. measurements between 2 and $45 \mathrm{MHz}$, using an interferometric technique to measure the velocity and the decay of the echo train to measure the damping. For the higher frequencies, we use Brillouin scattering at several scattering angles, using a five-pass Fabry-Perot interferometer with both $6471-\AA \mathrm{Kr}^{+}$and $5145-\AA \mathrm{Ar}^{+}$laser excitation. The speed of the sound is determined from the peak positions of the Brillouin doublet while the damping is determined from the full width at half maximum.

We plot the $\phi$-dependence of the speed of sound for several frequencies in Fig. 1. There is considerable dispersion in $v$ for each frequency, with the velocity initially decreasing slightly, then sharply increasing with volume fraction. However, there is an additional strong dispersion with frequency for volume fractions above 0.2 , with $v$ increasing further with frequency, $f$. To investigate this additional dispersion, we plot the frequency dependence of $v$ for several volume fractions in Fig. 2. The magnitude of the dispersion increases with increasing volume fraction. However, for all $\phi$, the dispersion occurs in the same frequency range, around $10^{8} \mathrm{~Hz}$. Furthermore, the data have reached 
their asymptotic limits at both the lowest and highest frequencies measured.

To account for these observations, we must consider the contribution of the micelles in the propagation of sound. The initial decrease in $v$ reflects the increased density of the suspension, since the density of the surfactant is greater than that of the oil. This results in an increase in the velocity of sound, which is inversely proportional to the square root of the average density of the mixture. By contrast, the increase in $v$ for $\phi \geq 0.2$ indicates an increase in the rigidity of the mixture. This must reflect the contribution of the close packed cores of the micelles, which must possess an increased rigidity. Alternatively, the adiabatic compressibility of the mixture decreases with increasing $\phi$, implying that the surfactant is less compressible than the oil. However, the increase in the velocity is higher at higher volume fractions, implying that the rigidity of the surfactant phase is increased at higher frequencies.

To explain this anomalous frequency dependence, we must consider the dynamics of the micelles. The micelles undergo Brownian diffusion, and on a time averaged basis, they behave as a random dispersion of independent spheres. However, the instantaneous configuration of the micelles can be significantly different. Because of the weak attractive interaction, the micelles will tend to form extended clusters whose size and concentration will increase with $\phi$. However, because the interaction between the micelles is rather weak, these clusters will last for only a short time, roughly determined by the interaction time, or the time during which the tails of neighboring micelles are entangled, $\tau_{c}$. At sufficiently high frequencies, $f>\tau_{c}{ }^{-1}$, the time scale of the measurement will be faster than the dynamics of this interaction and the micelle phase can be viewed as an instantaneous, connected network. Because the tail groups of the micelles that comprise this network are overlapping, it will support shear. This added shear modulus will increase the total fluid rigidity and hence the speed of sound. By contrast, at low frequencies, $f<\tau_{c}^{-1}$, the dynamics of the micelles will result in the networks being destroyed on time scales short compared the period of the sound, thereby relaxing any shear stress. Thus, the network is dynamic and the micelles behave as isolated spheres, with no additional increase in the rigidity due to a shear modulus of the surfactant phase. This provides a mechanism to account for the anomalous frequency dependence of the velocity of sound.

To quantify this hypothesis, we characterize the surfactant-oil mixture by its average complex elastic constant, $\beta^{*}=\beta^{\prime}+\mathrm{i} \beta^{\prime \prime}$, which is the inverse of the adiabatic compressibility associated with the longitudinal compression wave. This quantity is directly related to experimentally observable quantities: the speed of the sound is obtained from the real part, $v=\left(\beta^{1} / \rho\right)^{1 / 2}$, where $\rho$ is the average density. Similarly, the damping of the sound wave is determined from the imaginary part, with the attenuation length for the ultrasonic propagation given by $\alpha=f \beta^{\prime \prime} / 4 \pi \nu^{3} \rho$, and the width of the Brillouin peaks given by $\Delta v=f \beta^{\prime \prime} / 4 v^{2} \rho$. Although both experiments measure only the longitudinal sound propagation, this is also sensitive to the shear rigidity since $\beta^{*}=\kappa+4 \mu^{*} / 3$, where $\kappa^{*}$ and $\mu^{*}$ are the complex bulk and shear moduli respectively. Physically, when the material is compressed by a longitudinal stress, it resists strain in a direction transverse to the stress due to the shear modulus.

We use an effective medium model to calculate the average elastic constant of the mixture. It is determined from a volume-weighted mix of the elastic constants of each of the constituent phases. Thus, we attribute both an elastic constant, $\beta_{i}^{*}$, and a shear modulus, $\mu_{i}^{*}$, to each phase, the oil phase $(i=1)$, with volume fraction $1-\phi$, and the surfactant micelle phase $(i=2)$, with volume fraction $\phi$. To describe the $\phi$-dependent properties of the mixture at each frequency, we use a static effective medium model. This should be appropriate since the sound wavelength is always much larger than the micelle size. The dynamics of the micelles, and the resulting frequency dependence of the behavior, must be accounted for by the frequency dependence of $\beta_{2}{ }^{*}$ and $\mu_{2}^{*}$, the elastic constant and the shear modulus describing the surfactant micelle phase.

The parameters describing the oil phase, $\beta_{1} *$ and $\mu_{1}^{*}$, can be determined directly from experiment using the pure oil phase. Measurement of the sound velocity and damping determines $\beta_{1}^{\prime}$ and $\beta_{1}{ }^{\prime \prime}$. Furthermore, since a fluid cannot support a shear wave, $\mu_{1}{ }^{\prime}=0$, while $\mu_{1}{ }^{\prime \prime}=2 \pi f \eta$, where $\eta$ is the shear viscosity at $f=0$, which is measured independently. We cannot, however, independently measure any of the parameters for the second phase, as we cannot produce a sample comprised of pure AOT micelles. 


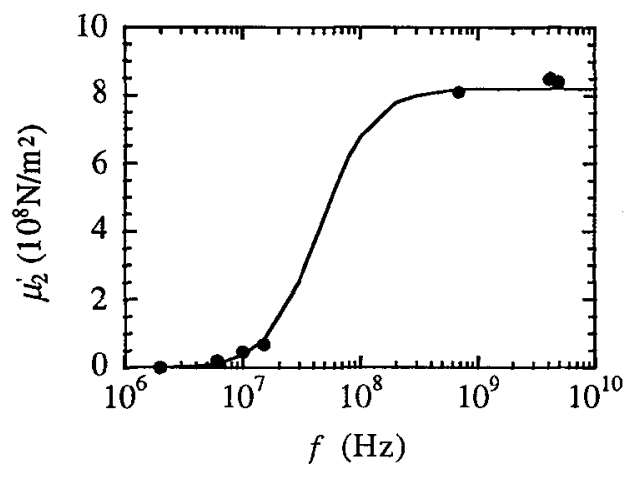

Fig. 3: Frequency dispersion of the real part of the shear modulus for the surfactant network phase obtained from the fit to the effective medium model for the micelles in decane. The solid line represents the form of a single-relaxation-time Debye model. description $[13,14]$ of the structure for the two components, assuming that both the oil and the micelles form bicontinuous, intermixed phases. The symmetric description requires the role of the lower volume fraction phase to be reversed as $\phi$ increases, and thus results in a percolation threshold. However, both components are assumed to be space filling. Since spheres cannot fill space, we multiply the effective medium volume fraction by 0.64 , corresponding to the volume fraction for random close packing of spheres [15]. To obtain agreement with the data requires the use of a non zero value for the shear modulus of the micelle phase, $\mu_{2}{ }^{*}$ in the fit, in accord with our physical picture. The solid lines through the data at $15 \mathrm{MHz}$ and $4 \mathrm{GHz}$ in Fig. 1 are examples of the excellent fits obtained.

The viscoelastic behavior of the mixture is reflected by the frequency dependence of $\beta_{2}{ }^{*}$ and $\mu_{2} *$. Indeed, the symmetric model used in fitting the high frequency data reduces exactly to Wood's formula when $\mu_{2} *=0$. The frequency dependence of the value of the real shear modulus of the micelles, $\mu_{2}^{\prime}$, obtained from the fits to the effective medium theories is shown in Fig. 3. It has roughly the same form of dispersion as the velocities, increasing at a fiequency of around $10^{8} \mathrm{~Hz}$ to a limiting value of $8 \times 10^{8} \mathrm{~N} / \mathrm{m}^{2}$ at the Brillouin frequencies. In addition, the bulk modulus of the micelle phase, $\kappa_{2}^{\prime}$, determined from the value of $\beta_{2}^{\prime}$ obtained from the fit, also exhibits a frequency dispersion. However, it decreases as $f$ increases, from $1.6 \times 10^{9} \mathrm{~N} / \mathrm{m}^{2}$ at $2 \mathrm{MHz}$ to $8 \times 10^{8} \mathrm{~N} / \mathrm{m}^{2}$ at $5 \mathrm{GHz}$. Physically, this may reflect the different properties of the micelle phase due to the time dependence of their correlations. Thus at low frequencies, $\kappa_{2}^{\prime}$ reflects a change in volume of the isolated micelles themselves. By contrast, at high frequencies, $\kappa_{2}$ ' reflects a change in volume of the random network of micelles, which can be accomplished by changing their relative positions. This requires a smaller force than changing the volume of the micelles themselves, resulting in a reduction of $\kappa_{2}^{\prime}$. This behavior is also reflected in a decrease in $\kappa^{\prime}$ at high frequencies for larger volume fiactions. The small values of $\mu_{2}^{\prime} \approx \kappa_{2}^{\prime} \approx 10^{9} \mathrm{~N} / \mathrm{m}^{2}$ imply that the micelle network is relatively soft, but nonetheless solid like.

For $\phi \leq 0.4$, the frequency dispersion of $v$ is reasonably well described by a single frequency Debye relaxation model, $v=v_{0}+\Delta v\left(\omega \tau_{c}\right)^{2} /\left[1+\left(\omega \tau_{c}\right)^{2}\right]$, where $v_{0}$ is the low frequency velocity, $\Delta v$ is the total velocity dispersion, and $\tau_{c}$ is the characteristic relaxation time. This behavior is shown by the solid line through the data in Fig. 2. At higher volume fractions, the velocity dispersion is no longer described as well using only one relaxation time. Nevertheless, the characteristic relaxation time for all the data is in the range of $\tau_{c} \approx 10^{-8} \mathrm{sec}$. The value for the real part of the shear modulus for the micelle network phase also displays a similar behavior with frequency. This is shown in Fig. 3, where we plot the value of $\mu_{2}$, obtained from a fit to the data for the velocity and damping, as a function of frequency. At the lowest frequencies, it is nearly zero, but then rises to a value of roughly $8 \times 10^{8} \mathrm{~N} / \mathrm{m}^{2}$ at the highest frequencies. The solid line through the data represents the same form of a single frequency Debye relaxation, illustrating that the behavior is similar to that observed for the dispersion of $v$ with frequency.

We emphasize that we are adopting a very simple picture to account for the anomalous frequency dispersion that is observed. At high frequencies, we assume that the sound wave probes the instantaneous configuration of the micelles, and that there is insufficient time for the networks of droplets to reconfigure their structure due to Brownian motion of the micelles. Thus the properties of the network are modeled by the static properties that a similar network would have at zero frequency, if the micelle configuration 
remained fixed. By contrast, at low frequencies, the dynamics of the micelles ensures that their configuration changes many time during the period of a sound wave, and thus they behave as independent, isolated spheres, even at higher volume fractions.

This system is an ideal one to use to measure the elastic properties of random, inhomogeneous mixtures. The fact that the micelles are dispersed in a fluid ensures a truly random dispersion of spheres. The fact that we can measure the speed of sound at both high and low frequencies ensures that we can distinguish the additional contribution of the rigidity of the micelle network. The difference between the high and low frequency moduli, $\Delta \beta^{\prime}$, is, to lowest order, linear in the change in $\mu_{2}^{\prime}$, and thus reflects the additional rigidity of the micelle network. We are able to discern a change in $v$ only in volume fractions above $\phi_{c} \approx 0.16$. Thus, we plot $\Delta \beta^{\prime}$ as a function of $\phi-\phi_{c}$ on a logarithmic plot in Fig. 4. The resolution of the Brillouin scattering measurements precludes measurements closer to $\phi_{c}$. Nevertheless, we observe a clear power-law behavior, $\Delta \beta^{\prime} \approx\left(\phi-\phi_{c}\right)^{\tau}$, with the critical volume fraction of $\phi_{c} \approx 0.16$ and a rigidity exponent of $\tau \approx 2.5$, as shown by the solid line in Fig. 4, which is a fit of this form to the data. This scaling extends over a rather large range in $\phi-\phi_{c}$, and is strongly suggestive of the behavior of a random percolating network. We emphasize that this observation is based solely on the experimental data, independent of any model. However, this is a dynamic rather than a static measurement. This limits the spatial extent of our sensitivity to the rigidity to roughly the wavelength of the sound, which is nonetheless at least 100 times the size of the micelles at the highest frequency. Finally, we also note that this rigidity transition occurs only for the surfactant micelle phase, which forms the random connecting network. The surrounding oil phase ensures that the entire mixture maintains a finite bulk modulus at all volume fractions, and does not itself exhibit a percolation threshold.

There are relatively few results available for elasticity percolation of random networks in three dimensions with which these data can be compared. No simulations or calculations for random multiply connected three dimensional networks exist. However, it is known that the electrical conductivity [16] scales with an exponent of $t \approx 2$ in three dimensions, with $\sigma=\left(\phi-\phi_{c}\right) t$. The elasticity exponent is expected to be the same as the conductivity exponent [17] provided only central forces exist between the constituent particles in the network [18]. By contrast, noncentral forces, or bond bending, break the similarity between conductivity and elasticity, leading to an increase in the elasticity exponent [19]. For singly connected networks [20], the exponent is predicted to be about 3.5. However, its value for networks in which multiple connections are possible, as is most likely the case here, is not know. Experimentally, measurements on beams of sintered metal [21] yielded $\tau \approx 3.8$ for the elasticity exponent while $t \approx 2.2$ for the conductivity exponent. Interestingly the scaling behavior for these beams also extended over a very large range in $\phi-\phi_{c}$, as is the case for our measurements. However, the exponent for the rigidity percolation for the micelle network is significantly different. This may reflect the different nature of the micelle interactions which may change $\tau$. Finally, the value of $\phi_{c}$ found here is consistent with that found from simulations of interacting spheres, and is in the same range as is observed for the conductivity percolation for these systems [22].

One of the key parameters which determines the unique behavior observed in this colloidal system is the magnitude of the attractive interaction energy between two micelles, $E_{a}$. Variations in $E_{a}$ will result in strong variations in the characteristic time of the interactions, $\tau_{c}$, and hence the frequency at which the dispersion in the velocity is observed. Increasing the value of $E_{a}$ will result in an increase in the interactions time, $\tau_{c}$, or a corresponding decrease in $f_{c}$. The value of $E_{a}$ is very sensitive on the chain length of the alkane used as the solvent [12]. As the chain length is increased, the interaction energy also increases. The consequences of these changes on the propagation of sound are summarized by the data shown in Fig. 5, where we plot the $\phi$ dependence of $v$ measured at different frequencies for micelles in solvents consisting of three different linear alkanes: hexane $\left(\mathrm{C}_{6} \mathrm{H}_{14}\right)$, decane $\left(\mathrm{C}_{10} \mathrm{H}_{22}\right)$ and hexadecane $\left(\mathrm{C}_{16} \mathrm{H}_{34}\right)$. The results at 2 and $15 \mathrm{MHz}$ were obtained using ultrasonic measurements while those at 5 GHz were obtained with Brillouin scattering. The sound velocity increases for each data set for different solvents, reflecting the increased velocity in the solvent itself. The data for decane is identical to that 


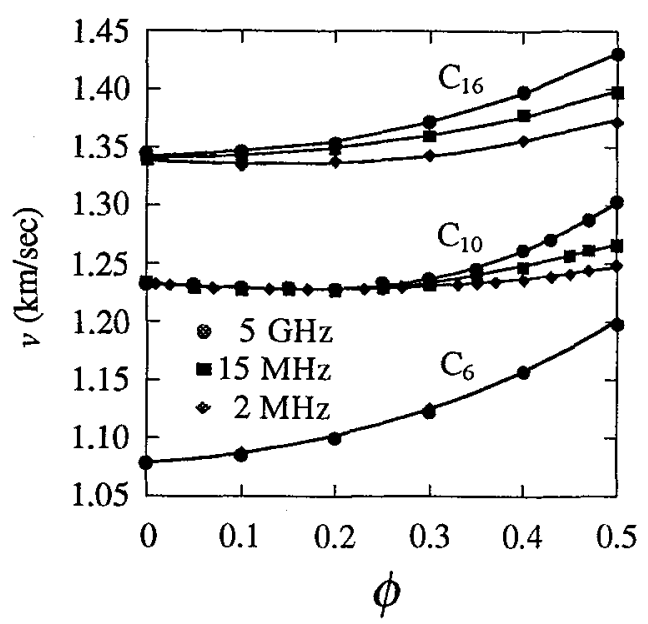

Fig. 5: Velocity of sound for AOT micelles as a function of volume fraction for several different solvents: Hexane $\left(\mathrm{C}_{6}\right)$, decane $\left(\mathrm{C}_{10}\right)$ and hexadecane $\left(\mathrm{C}_{16}\right)$. Ultrasonic techniques were used to obtain the data at $2 \mathrm{MHz}$ and $15 \mathrm{MHz}$, while Brillouin scattering was used to obtain the data at 5 $\mathrm{GHz}$. The solid lines are fits to the effective medium theories.

exhibits no additional frequency dispersion for all volume fractions. Moreover, the fit to the data shown is obtained without including a shear modulus for the micelle phase in the effective medium model. Thus, $f_{c}$ must be greater than all of our experimentally accessible frequencies, consistent with the decrease in $E_{a}$ for hexane. The data for hexadecane exhibits additional frequency dispersion for all $\phi$. Moreover, a real component for the shear modulus of the surfactant phase must be included in the effective medium model to obtain the fits to the data shown. Thus, now $f_{c}$ has been decreased below our experimentally accessible frequency range by the large increase in $E_{a}$. We conclude that the behavior as the solvent is changed is consistent with our physical picture of the dynamics of the system.

Another way to change the strength of the interaction is to swell the micelles with water, and make microemulsions. The increased radius of the microemulsion droplets will increase the contact area of the surfactant molecules, thereby increasing $E_{a}$. There will, however, be a competing effect. Since the droplets are being swollen with water, which can not support shear the way the surfactant shells do, the intrinsic shear modulus of the network should be expected to decrease as the size of the droplets grows. To investigate these effects, we show, in Fig. 6, the results of $v$ as a function of $\phi$ for microemulsions in decane, using droplets with three different radii, 25,45 and $75 \AA$. The data were obtained at a frequency of $5 \mathrm{GHz}$ using Brillouin scattering. The velocities of each of the three systems increase with increasing $\phi$. The lines through the data represent the fits obtained using the effective medium model. For the two microemulsions with the smaller radii, the data must be fit by including a real part of the shear modulus for the droplet network phase. However, the value of the shear modulus for the network phase obtained from the fits decreases as the droplet size grows. Moreover, the fit to the data for the largest size of microemulsions is obtained without including any shear modulus in the theory. Thus this behavior is in accord with our physical expectations.

We can also investigate the frequency dispersion of the sound velocity in these microemulsions. We determine the dependence of $v$ on $\phi$ using ultrasonic measurements from 2 to $30 \mathrm{MHz}$. Virtually no additional frequency dispersion is observed for all volume fractions for any of the samples. These observations suggest that the contact area for all three sizes has been increased leading to a larger $E_{a}$, and hence an increased $\tau_{c}$ Thus $f_{c}$ is decreased below the values probed by our experiments and no additional frequency dispersion is observed. Thus, again this behavior is in accord with our physical expectations.

To investigate the generality of the dynamic rigidity percolation observed in the micelles, we can look for similar behavior in the microemulsions. To do this, we can exploit the behavior observed in the 


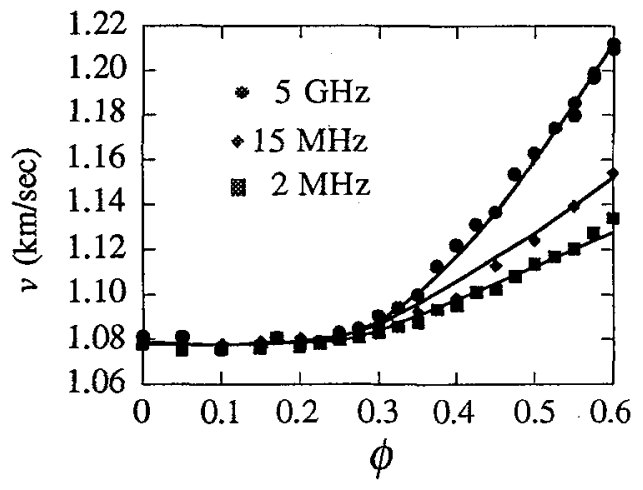

Fig. 7: Velocity of sound in $25-\AA$ radius AOThexane-water microemulsions as a function of volume fraction for different frequencies. The solid lines represent fits to the effective medium model, treating the droplet phase as a random, connected medium that can support shear.

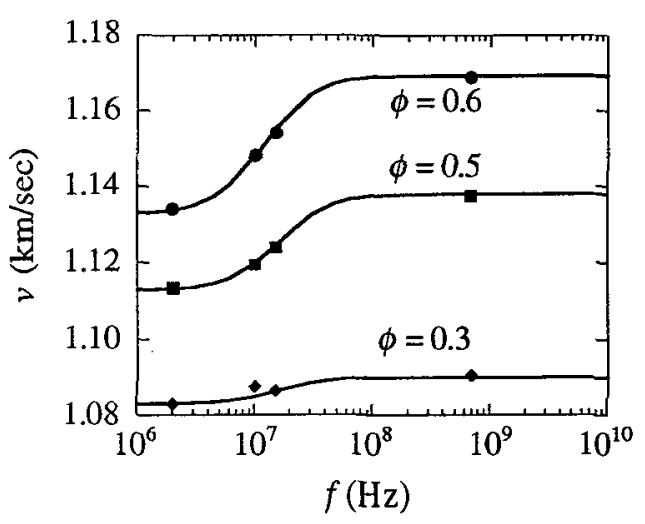

Fig. 8: Frequency dispersion of the sound velocity of $25-\AA$ radius microemulsions in hexane at three different volume fractions: $\phi=0.3,0.5$ and 0.6 . The solid lines are single-relaxation-frequency Debye forms.

micelles and change the interaction energy by changing the chain length of the hydrocarbon solvent. In order to bring the characteristic frequency into the experimentally accessible range, we must decrease the interaction energy, which requires the use of a shorter solvent molecule. However, we must also use microemulsions with a smaller radius to ensure that the intrinsic shear modulus of the droplets themselves is sufficiently high. Thus we use the $25 \AA$ radius droplets, made in hexane. The results are shown in Fig. 7 , where we plot the sound velocity as a function of droplet volume fraction for three different frequencies. Considerable additional frequency dispersion is observed, and the behavior is very similar to that seen with the micelles in decane, consistent with our expectations. The effective medium model used to obtain the fit to the data shown for the $2 \mathrm{MHz}$ results requires the use of shear modulus very nearly zero. By contrast, the fits to the data at the higher frequencies require the use of larger values of the shear modulus. We also show, in Fig. 8, the frequency dispersion of the velocities for three different volume fractions. Again, the results obtained are strikingly similar to those obtained for the micelles in decane. The solid lines through the data again reflect a single frequency Debye relaxation model for the results, with a frequency of $f_{c} \approx 10^{8} \mathrm{~Hz}$.

The similarity in the behavior between the $25 \AA$ radius microemulsions in hexane and the micelles in decane also extends to the scaling behavior of the increase in the modulus with volume fraction. This is shown in Fig. 9, where we plot $\Delta \beta^{\prime}$, as determined from the increase in sound velocity between the lowest and the highest frequencies, as a function of $\phi-\phi_{c}$. Scaling behavior is again observed, with $\Delta \beta^{\prime} \sim\left(\phi-\phi_{c}\right)^{\tau}$, where $\phi_{c} \approx 0.15$ and $\tau \approx 2.5$. These results are in excellent agreement with those obtained for the micelles in decane. Thus, we conclude that dynamic rigidity percolation is a very general form of behavior for these systems. It is interesting to note, however, that the value of the critical volume fraction for percolation does not change much for the two cases. We might expect $\phi_{c}$ to be sensitive to the interaction energy between the droplets, and thus to vary with the systems. Presumably the interaction energies in the two cases examined are very nearly the same, accounting for the similarity in the values of $\phi_{c}$. Further experiments to determine the sensitivity of $\phi_{c}$ on $E_{a}$ would be of great interest.

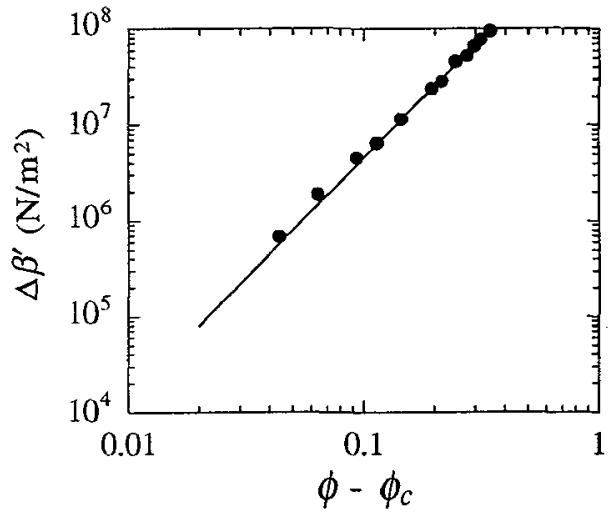

Fig. 9: Critical scaling behavior of the additional modulus of the droplet network phase of the $25-\AA$ radius microemulsions in hexane at high frequencies, when it forms a random rigid network. The exponent is $\tau \approx 2.5$ and the critical volume fraction is $\phi_{c} \approx 0.16$. 


\section{HARD SPHERE COLLOIDS}

All of the results discussed in the previous section explored the behavior of a colloidal system where the size of the individual particles are substantially smaller than the size of the wavelength of the sound. We now examine the sound propagation in a colloidal system where the size of the particles is comparable to the wavelength of the sound $[7,8]$. The system comprises monodisperse polymethylmethacrylate (PMMA) spheres immersed in a mixture of dodecane and carbon disulfide where the relative concentration of the two solvents has been adjusted to nearly perfectly match the index of refraction of the colloidal particles, thereby eliminating any potential problems of multiple scattering of light. The colloidal particles are sterically stabilized by a thin layer of grafted polymer with a thickness of about $15 \mathrm{~nm}$, much smaller than the diameter of the particles themselves. The interaction between the particles is very well approximated as that of hard spheres, and they exhibit a very rich phase behavior as the particle volume fraction is changed [23]. At low $\phi$, the colloidal particles behave as a fluid, with short range correlations between the colloidal particles that are fluid-like. At larger volume fractions, $\phi>0.49$, the colloidal particles form either a colloidal glass or a colloidal crystal.

Here, we study the propagation of sound through this system using Brillouin scattering. Although there is very little scattering of light by this colloidal system, because of matching of the indices of refraction of light, there is still considerable scattering of sound by the colloidal particles, as neither the sound velocities, nor the impedances, are matched. By changing the scattering angle, we can vary the scattering wave vector, and hence the wavelength of the sound. We are able to make measurements ranging from very small angles, where $q d<2 \pi$, to larger angles, where $q d \approx 1$, to even larger angles, where $q d>1$. We can thus investigate the propagation of sound as the wavelength becomes comparable to the size of the scatterers.

The propagation of sound through a granular material is one of the most important probes of the nature of the material, since the structure and correlations between the grains can have a profound effect on the acoustic properties. A suspension of hard spheres represents one of the simplest and most fundamental granular systems for investigating sound propagation [24]. Since the continuous phase is a fluid, which can not support shear, there is no long range rigidity in the medium. Thus, in the long wavelength limit, it can be rigorously shown that there is only one propagating longitudinal mode [14]. This behavior has long been expected to persist to the shorter wavelength limit [24]. However, we show here that this expectation is invalid, and that as the wavelength becomes comparable to the size of the spheres, a second, unexpected, longitudinal mode also exists. The origin of this mode is attributed to a new surface excitation that exists only for solid particles which possess a shear modulus and only when the wavelength of the sound approaches the diameter of the hard spheres. In this case, this new acoustic excitation can propagate coherently from sphere to sphere provided the volume fraction of the spheres is sufficiently large. As the sphere diameter becomes much larger than the wavelength of sound, this excitation becomes a Stoneley wave, an acoustic excitation that is confined to a flat interface between a fluid and a solid [25]. In this paper, we present the full dispersion curves for the longitudinal acoustic excitations of suspensions of hard sphere colloids as the volume fraction is varied.

We use two samples of PMMA spheres, each of different diameter. The first sample has a diameter of $d=340 \mathrm{~nm}$, while the second has a diameter of $d=650 \mathrm{~nm}$, where in both cases we quote the diameter of the core, without the stabilizing polymer. To compare the results for the different spheres, and to compare the wavelength of the sound to the diameter of the spheres, we plot all the results in terms of the dimensionless parameter $q d$. The static structure factor for each of the suspensions is determined with static light scattering to confirm the range of $q d$ available in each case. The smaller spheres are more useful for exploring the range where the wavelength of the sound is comparable to the diameter of the spheres, or when $q d \approx 2 \pi$, while the larger spheres are more useful for studying the regime where the wavelength of sound is much smaller than the sphere diameter, or when $q d$ » $2 \pi$.

In the limit of very low scattering angles, when the wavelength of the sound is much larger than the sphere size, we expect to observe hydrodynamic behavior, with only a single propagating longitudinal sound mode. The lowest value of $q d$ that we can reach is $q d \approx 1$, obtained using the smaller spheres with a scattering angle of $9^{\circ}$. In Fig. 10, we show the Brillouin spectra obtained at a scattering angle of $16^{\circ}$ corresponding to $q d \approx 1.7$. We show data for three different sphere volume fractions, $\phi=0.16,0.38$ and 0.51 . For the lowest volume fraction, the Brillouin peak is almost unchanged in shape and position from that observed in the index matching fluid with no spheres present. However, the intensity of the Rayleigh peak is increased by several orders of magnitude with the addition of the spheres, while the intensity of the Brillouin peak is virtually unchanged. As $\phi$ increases, the Brillouin peaks shift to larger frequencies and become broader. This behavior can be accounted for by the increase in the volume fraction of the solid spheres, which have a larger velocity of sound than the fluid. In fact, the $\phi$ dependence of the 


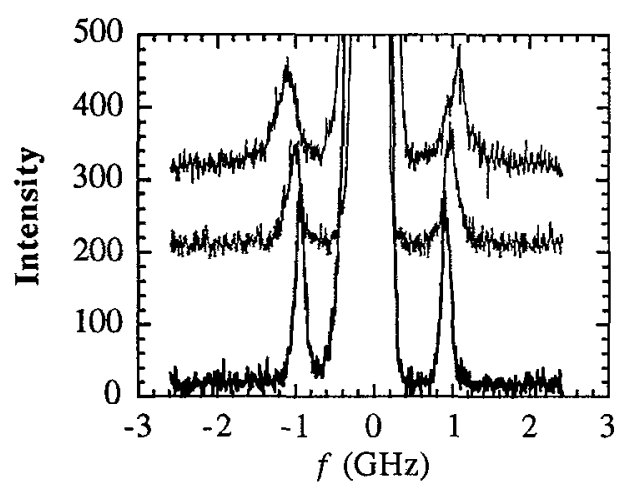

Fig. 10: Brillouin spectra for three different volume fractions of $340 \mathrm{~nm}$ diameter PMMA spheres measured at $q d \approx 1.7$, in the hydrodynamic, or long wavelength regime of propagation of acoustic waves.

velocity of sound at this value of $q d$ is well described using the simple effective medium model discussed in the previous section, without including any shear modulus.

As $q d$ approaches $\pi$, a qualitative change is observed in the Brillouin spectra. The peaks no longer increase in frequency as the volume fraction is increased. Moreover, a second, very low intensity, peak appears at higher frequency. This peak persists and grows in intensity as $q d$ is increased. Typical spectra obtained at $q d \approx 2.8 \pi$ are shown in Fig. 11 for three different volume fractions. For the lowest volume fraction, $\phi=0.16$, only one mode can be clearly resolved, although its shape is somewhat asymmetric, suggesting that two modes may exist, but with such small difference in frequency that they can not be independently resolved. At the higher volume fractions, $\phi=0.38$ and 0.51 , the two modes are both clearly resolved. Both peaks correspond to longitudinal modes, as confirmed by the absence of any depolarized scattering.

We plot the full dispersion curves for the $340 \mathrm{~nm}$ diameter spheres for the same three volume fractions, $\phi=0.16,0.38$ and 0.51 , in Fig. 12. For comparison, we also show the linear dispersion curves for the longitudinal sound modes that would exist in the pure phases. The solid line corresponds to solid PMMA, while the dashed line corresponds to the pure index matching fluid. At low $q d$, only a single mode is observed for all values of $\phi$. The dispersion for each volume fraction is linear as $q d$ approaches zero. The frequencies of this mode increase with increasing $\phi$. However, as $q d$ increases further, the dispersion curves for all three volume fractions begin to flatten and the frequency of the mode for the higher volume fractions actually drops below that of the lower volume fractions. At $q d \approx \pi$, the second mode appears for volume fractions above $\phi=0.16$. This mode persists as $q d$ becomes larger, and the splitting between the two modes increases with increasing volume fraction. However, for $\phi \leq 0.16$, the frequency of the single mode is virtually indistinguishable from that of the index matching fluid. For the higher volume fractions, which possess two distinct modes, the frequency of the faster mode lies in between those of the pure PMMA and pure index matching fluid. Moreover, the frequency of this mode increases with increasing volume fraction. This mode is
Fig. 12: The dispersion curves for the longitudinal acoustic modes for three different volume fractions of the $340 \mathrm{~nm}$ diameter spheres, plotted as a function of $q d .$. The solid line represents the dispersion curve for the pure PMMA while the dashed line represents the dispersion curve for the pure index matching fluid.

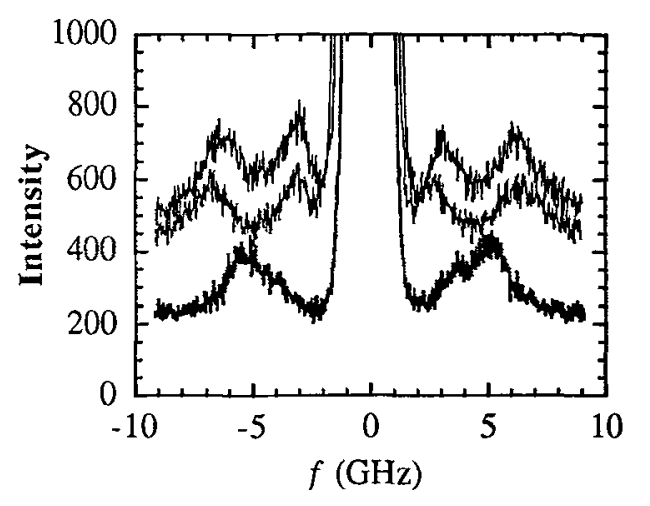

volume fractions of $340 \mathrm{~nm}$ diameter PMMA spheres measured at $q d \approx 2.8 \pi$. Two distinct modes are clearly observable for the two higher volume fractions.

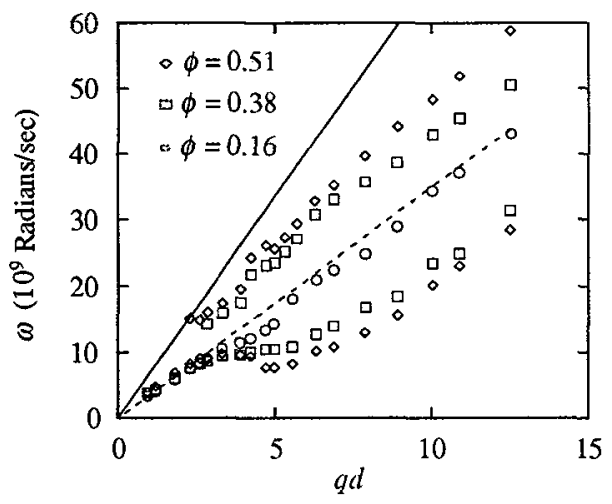




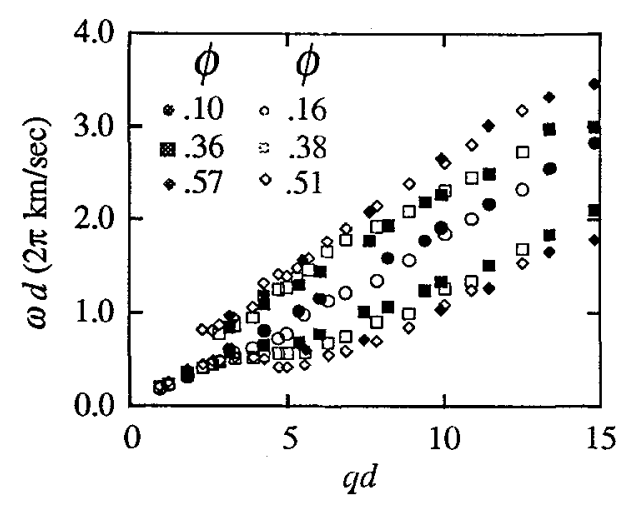

Fig. 13: Scaled dispersion curves for the 340 and $650 \mathrm{~nm}$ diameter spheres for different volume fractions. The solid symbols refer to the larger spheres while the open symbols refer to the smaller spheres.

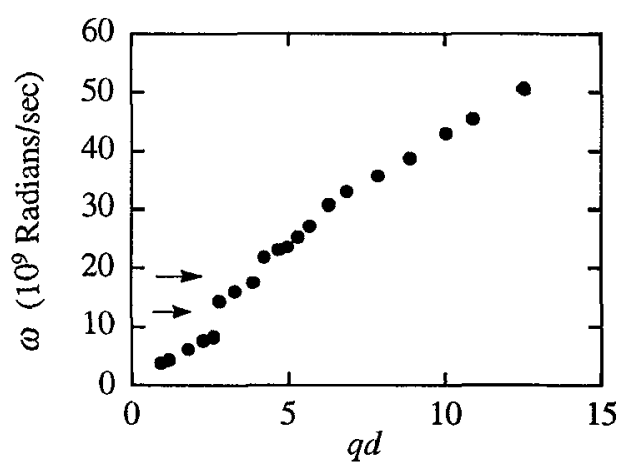

Fig. 14: The dispersion curve of the high frequency mode for the $340 \mathrm{~nm}$ diameter spheres at a volume fraction of 0.38 . the arrows correspond to the frequencies of the peaks of the scattering amplitudes of longitudinal acoustic waves incident on a single, isolated sphere immersed in a fluid.

consistent with the behavior expected for a mode in a composite medium. By contrast, the frequency of the slower mode lies below those of either of the two pure materials that make up the system. Moreover, its frequency decreases as the volume fraction increases. Finally, both modes soften for all volume fractions around the peak in the static structure factor, $q d \approx 2 \pi$. The softening of the lower frequency mode is considerably more pronounced and the degree of softening increases with volume fraction.

The behavior of the dispersion curves is not unique to this set of spheres. Very similar behavior is observed for the larger spheres. Again two independent modes are observed for $q d$ above $\pi$, and the trends with volume fraction for each of the modes are the same as shown in Fig. 12 for the smaller spheres. The similarity of the data for the different sets of spheres is emphasized in the plot shown in Fig. 13, where we plot the dispersion curves for both sizes, but scaled by their diameters. Thus both sets are plotted as functions of $q d$, and the frequencies of each mode have also been scaled by the sphere diameter. The vertical axis could be made dimensionless by normalizing by a velocity, such as the average velocity of sound in the medium. In Fig. 13, the open data points represent data collected with the smaller spheres and are the same as those plotted in Fig. 12. The solid points represent data collected with the larger spheres, for volume fractions of $\phi=0.10,0.36$ and 0.57 . As can be seen, the data for similar values of $\phi$ scale together quite well, confirming the generality of these results.

Perhaps the most surprising feature of these results is the existence of two, rather than one, longitudinal acoustic modes. To account for the origin of this behavior, we consider the nature of each of the modes individually. The higher frequency mode has the dependence on volume fraction expected for an acoustic excitation propagating through a composite medium: the frequency increases as the volume fraction of solid increases. Since the velocity of sound is much greater in the pure solid than in the pure fluid, the frequency of an acoustic excitation propagating through the two media should also increase. Furthermore, the frequency of this mode lies between those of the two pure materials. Thus, we attribute the higher frequency mode to an acoustic excitation that propagates through both the fluid and the solid. Moreover, since the single excitation observed at low $q d$ also has the same dependence on volume fraction, and is so well described by an effective medium model that assumes that it propagates through the two materials, it too must be associated with the acoustic excitation propagating through the liquid and the solid spheres.

The nature of the propagation of this mode is strongly influenced by the presence of the spheres. While the index of refraction of the fluid and the solid are very nearly matched, eliminating any strong scattering of light from the system, this is not the case for sound. There is a large mismatch in the impedance for sound to propagate from one material to the other, and as a result, the sound waves are strongly scattered from the interfaces. The consequence of this is most clearly observed by examining the dispersion curve for this excitation for a single volume fraction. As an example, we plot, in Fig. 14, the dispersion curve for the higher frequency mode for the smaller spheres with $\phi=0.38$. Two gaps in the dispersion curve are clearly evident at frequencies of $\omega \approx 10$ and $20 \times 10^{9} \mathrm{radians} / \mathrm{sec}$, corresponding to frequencies where the excitation does not propagate. Moreover, the dispersion curve becomes flat on either side of the gaps. The frequencies of these gaps correspond to the frequencies calculated for the 
peaks in the scattering amplitude of a longitudinal wave incident on a single sphere immersed in a fluid, which are shown by the two arrows. Thus when the scattering from the isolated spheres becomes very large, the wave can no longer propagate, and gaps develop in the dispersion curve. The large increase in the scattering from the individual spheres results from the excitation of internal resonances within the spheres. There are a large number of these resonances at higher frequencies, with the two gaps observed corresponding to the two lowest frequency resonances. Gaps in the dispersion curve at higher frequencies may also be discernible, but not as clearly. While sound at these frequencies can not propagate with the wave vectors corresponding to this mode, it can propagate at another wave vector, as the lower frequency mode.

The key to the origin of the lower frequency mode is its very slow velocity, as it propagates more slowly than a longitudinal wave in either of the two pure materials. The only mode that can propagate in a system comprised of a solid and a fluid at a velocity that is slower than the longitudinal velocities in both materials is a Stoneley wave. This is a propagating wave confined to the interface between a solid and a fluid, and is analogous to a Raleigh wave, which is a propagating wave confined to the interface of solid and vacuum [25]. The existence of a Stoneley wave requires a shear modulus in the solid. It consists of both longitudinal and transverse polarizations in the solid and a purely longitudinal component in the fluid. The magnitude of the oscillation decays exponentially away from both of the surfaces, ensuring the confinement of the excitation to the interface. While a Stoneley wave is a solution to the elastic equations that is confined to a flat interface, the excitation here is initially observed when the wavelength of the sound is less than the size of a single sphere. Moreover, from the width of the Brillouin peaks, we can ascertain that the excitation propagates over length scales larger than a single sphere. Thus this excitation must propagate coherently from sphere to sphere. This presumably occurs through the exponentially decaying portions of the excitation in the fluid. If another sphere is nearby, the excitation can hop between the two spheres. This would account for the fact that this new mode is only observed when the volume fraction of the spheres is relatively large, ensuring that there are neighboring spheres close by. Moreover, this would account for the very unusual dependence of the velocity on volume fraction. At higher volume fractions, there are more interfaces relative to the amount of fluid. The velocity of the excitation on the interfaces is lower than in the fluid, thus leading to the measured decrease in the velocity as the volume fraction of spheres increases.

Support for our interpretation comes from the dispersion curves measured for the larger spheres, which span a greater range in $q d$. Rather than plot the frequency as a function of $q d$, in Fig. 15 , we show the phase velocities, $v=\omega / q$ of the two modes for several volume fractions of the larger spheres, $\phi=0.10,0.36$ and 0.57 . The velocity of the single mode observed for the lowest volume fraction is almost indistinguishable from that of the index matching fluid, at $1.18 \mathrm{~km} / \mathrm{sec}$. The velocity of the lower frequency mode is initially quite slow, and decreases near $q d \approx 2 \pi$. However, as $q d$ increases to much larger values, the velocity increases somewhat, and then approaches an asymptotic value, which is the same for all volume fractions. At these large values of $q d$, the interface appears very nearly flat on the scale of a wavelength. The velocity of this mode measured at high $q d$ is $0.86 \mathrm{~km} / \mathrm{sec}$, which corresponds directly to the velocity calculated for a Stoneley wave at a flat interface between PMMA and the index matching fluid. By contrast, the velocity of the higher frequency mode seems decrease and asymptotically approach the velocity of the index matching fluid for all volume fractions of spheres. This is again consistent with an effective medium picture for this excitation. At these very large values of $q d$, it is possible to fit many wavelengths of the excitation in either a single sphere or in the fluid between the spheres. Thus it is possible to detect the excitations within each of the pure materials that comprise the suspension. The higher

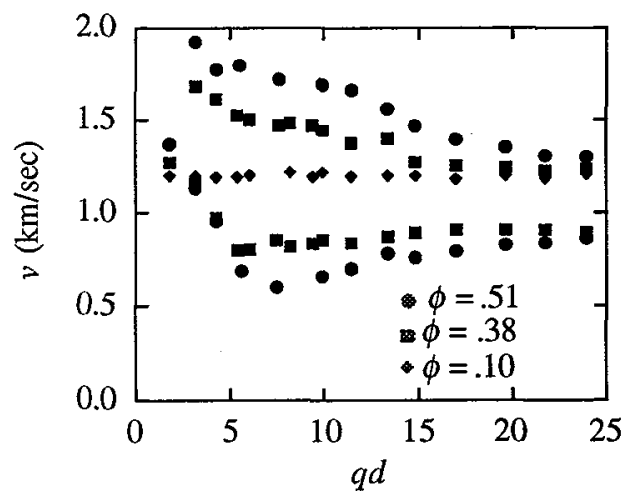

Fig. 15: The phase velocities for three different volume fractions of the $650 \mathrm{~nm}$ diameter spheres, plotted as a function of $q d$. The velocity of the single mode present for the lowest volume fraction is nearly indistinguishable from that of the liquid, $1.18 \mathrm{~km} / \mathrm{sec}$. The velocity of the higher frequency mode reaches a peak around $q d \approx \pi$, then decreases as $q d$ increases, ultimately asymptotically approaching that of the pure fluid for both volume fractions. The velocity of the lower frequency mode decreases until $q d \approx 2 \pi$, then increases, asymptotically approaching a value of $0.860 \mathrm{~km} / \mathrm{sec}$ for both volume fractions, corresponding to the velocity of a Stoneley wave at a flat interface between solid PMMA and the index matching fluid. 
frequency excitation therefore corresponds to the longitudinal sound wave within the pure fluid. In fact, at the very highest values of $q d$, a new excitation is visible in the spectrum when recorded with an increased free spectral range of the interferometer. This new peak has a phase velocity corresponding very nearly to that of a longitudinal sound wave in pure PMMA. These observations lend very strong support to our interpretation of the results.

Further support for these observations comes from a theoretical calculation of the density of states as a function of both frequency and wave vector using an effective medium model $[26,27]$. The system is modeled as a solid sphere surrounded by a coating of fluid and immersed in a continuum that reflects the properties of the rest of the system. The full elastic wave equation is solved self-consistently in this system to obtain the density of acoustic states. The peaks in the density of states correspond to the acoustic modes observed in the Brillouin scattering measurements. Excellent agreement is found between the peaks in the calculated density of states and the frequencies of the modes observed experimentally. Moreover, the widths of the peaks in the calculation are in good agreement with the measured widths of the modes. Two modes are observed in the theory, corresponding to the two modes observed experimentally. Even the gaps in the dispersion curve of the higher frequency mode are accurately reproduced in the calculation. Furthermore, in the calculation, the volume fraction of the system is adjusted by varying the thickness of the fluid coating. Again excellent agreement is obtained with the experimental data as the volume fraction is varied. Finally, it must be emphasized that all the parameters required for input to the effective medium theory are known independently so that there are no free fitting parameters in comparing the data to the results of the theory. Thus the excellent agreement with the data provides very strong confirmation of the validity of the theory, and of the validity of our interpretation of the physics.

\section{CONCLUSIONS}

We have presented the results of a study of sound propagation in two different colloidal systems. In the first case, consisting of both micelles and microemulsions in an oil continuous phase, the size of the colloidal particles is always substantially smaller than the wavelength of the sound. In the second case, consisting of PMMA spheres suspended in an index matched fluid and interacting through the hard sphere potential, the wavelength of the sound is varied relative to the size of the colloidal particles, from much greater than, to comparable to, and finally to much smaller than, the particle diameter. In both cases, new physics is observed.

In the case of the surfactant system, we observe an anomalous frequency dispersion in the velocity only at higher volume fractions of droplets. The increase in modulus of the system scales with the increase in volume fraction above some critical value, $\Delta \beta^{\prime} \sim\left(\phi-\phi_{c}\right)^{\tau}$, where $\phi_{c} \approx 0.16$, and $\tau \approx 2.5$. The origin of this behavior is interpreted in terms of a dynamic rigidity percolation. Since there is a small attractive interaction between the droplets, they will tend to form clusters that will last for a very small time. However, if the period of the sound wave is smaller than the characteristic time during which the clusters remain intact, the acoustic propagation will be sensitive to the properties of the random networks of the clusters. These networks can support shear if they span the system and thus can increase the bulk modulus of the medium resulting in the observed increase in the sound velocity at higher frequencies and for volume fractions above $\phi_{c}$.

In the case of the hard spheres, we observe a new acoustic excitation, in addition to the excitation expected for a composite granular system. This new acoustic excitation possesses very unusual properties. Its phase velocity is lower than those for either of the pure materials which make up the system. Moreover, its velocity decreases as the volume fraction of solids is increased. We interpret the origin of this anomalous excitation as resulting from a new interface mode. At high $q d$, this mode reverts to a Stoneley wave, a propagating longitudinal mode that is confined to the interface of a fluid and a solid. Our results show that this interfacial wave also exists, in a modified form, when the solid is a sphere of diameter comparable to the wavelength of the excitation. In this case, the mode can coherently propagate between adjacent spheres through the decaying longitudinal portion of the excitation in the fluid, provided the neighboring spheres are sufficiently close. Thus, we only observe this new mode when the volume fraction of spheres is greater than about 0.2 .

The results presented in this paper illustrate some of the rich new physics that is observed in the propagation of sound in colloidal systems. These measurements probe the dynamics of the system. They are sensitive to the bulk compressibility of the medium, in contrast to static light scattering measurements, which typically are sensitive to the osmotic compressibility of the colloidal particles. As such the study of sound propagation adds new and different information to our understanding of complex colloidal fluids. 
We gratefully acknowledge the assistance of Shobo Bhattacharya and Mark Higgins in the ultrasonic measurements and Peter Pusey for providing the PMMA samples.

\section{REFERENCES}

1. Ye, L., Weitz, D.A., Sheng, P., Bhattacharya, S., Huang, J.S. and Higgins, M.J., Phys. Rev. Lett. 63 (1989) 263.

2. Ye, L., Weitz, D.A., Sheng, P. and Huang, J.S., Phys. Rev. 44 (1991) 8249.

3. Eckwell, P., Mandell, L. and Fontell, K., J. Colloid Interface Sci. 33 (1970) 215.

4. Jean, Y.C. and Aacke, H.J., J. Am. Chem. Soc. 100 (1978) 6320.

5. Zulauf, M. and Eicke, H.F., J. Phys. Chem. 83 (1979) 480.

6. Assih, T., Larche, F. and Delord, P., J. Colloid Interface Sci. 89 (1982) 35.

7. Liu, J., Ye, L., Weitz, D.A. and Sheng, P., Phys. Rev. Lett. 65 (1990) 2602.

8. Weitz, D.A., Liu, J., Ye, L. and Sheng, P., Phonon Dispersion in Suspensions of Hard Sphere Colloids, in Physical Phenomena in Granular Materials, Cody, G.C., Geballe, T. and Sheng, P., Editors. (Materials Research Society, Pittsburgh, 1990) p. 93.

9. Kotlarchyk, M. and Huang, J.S., J. Phys. Chem. 89 (1985) 1985.

10. Kotlarchyk, M., Chen, S.H. and Huang, J.S., J. Phys. Chem. 86 (1982) 3273.

11. Kotlarchyk, M., Huang, J.S. and Chen, S.-H., J. Phys. Chem. 89 (1985) 4382.

12. Huang, J.S., J. Phys. Chem. 82 (1985) 480.

13. Sheng, P., in Homogenization and Effective Moduli of Materials and Media, Ericksen, J.L., Kinderlehrer, D., Kohn, R. and Lions, J.-L., Editors. (Springer-Verlag, New York, 1986) p. 196.

14. Hashin, Z., J. Appl. Mech. 29 (1962) 143.

15. Zallen, R., The Physics of Amorphous Solids. ed. (Wiley, New York, 1983).

16. Stauffer, D., Introduction to Percolation Theory. (Taylor and Francis, London, 1985).

17. DeGennes, P.G., J. Phys. Lett. 37 (1976) L1.

18. Alexander, S., J. Phys. (Paris) 45 (1984) 1939.

19. Feng, S., Sen, P., Halperin, B.I. and Lobb, C.J., Phys. Rev. B 30 (1984) 5386.

20. Kantor, Y. and Webman, I., Phys. Rev. Lett. 52 (1984) 1891.

21. Deptuk, D., Harrison, J.P. and Zawadzki, P., Phys. Rev. Lett. 54 (1985) 913.

22. Safran, S.A., Webman, I. and Grest, G.S., Phys. Rev. A 32 (1985) 506.

23. Pusey, P.N. and Megan, W.v., Nature 320 (1986) 340.

24. Schwartz, L.M. and Plona, T.J., J. Appl. Phys. 55 (1984) 3971.

25. Brekhovskikh, L.M., Waves in Layered Media. (Academc Press, New York, 1980).

26. Jing, X.D., Sheng, P. and Zhou, M.Y., Phys. Rev. A in press.

27. Jing, X.D., Sheng, P. and Zhou, M.Y., Phys. Rev. Lett. 66 (1991) 1240. 